\title{
On Regular Pre-Semiclosed Sets in Topological Spaces
}

\author{
T. Shyla Isac Mary ${ }^{*}$ and P.Thangavelu2 \\ 1 Department of Mathematics, Nesamony Memorial Christian College, \\ Martandam- 629165, India \\ 2 Department of Mathematics, Aditanar college, Tiruchendur-628216, India \\ *E-mail: shylaisaacmary@yahoo.in
}

Received April 15, 2010 / Accepted May 18, 2010

\begin{abstract}
The generalized closed sets in point set topology have been found considerable interest among general topologists. Veerakumar introduced and investigated pre-semi- closed sets and Anitha introduced pgprclosed sets. In this article the concept of regular pre-semiclosed sets is introduced in topological spaces and its relationships with other generalized sets are investigated .
\end{abstract}

Keywords: pre-semiclosed, pgpr-closed, semi-preclosure, rg-open and g-open sets.

\section{Introduction}

Levine[9] introduced generalized closed (briefly g-closed) sets in topology. Researchers in topology studied several versions of generalized closed sets. In this paper the concept of regular pre-semiclosed (briefly rps-closed) set is introduced and their properties are investigated. This class of sets is properly placed between the class of semipreclosed sets and the class of pre-semiclosed sets. Certain preliminary concepts are given in the section 2 , the concept of rps-closedness is studied in section 3 and the reference is given at the end followed by a diagram that gives the relationships among the generalized closed sets in topological spaces. 


\section{Preliminaries}

Throughout this paper $X$ and $Y$ represent the topological spaces on which no separation axioms are assumed unless otherwise mentioned. For a subset $A$ of a topological space $X$, clA and int $A$ denote the closure of $A$ and the interior of $A$ respectively. $X \backslash A$ denotes the complement of $A$ in $X$. Throughout the paper $\square$ indicates the end of the proof. We recall the following definitions.

\section{Definition 2.1}

A subset $A$ of a space $X$ is called

(i) pre-open [12] if $A \subseteq$ int clA and pre-closed if $c l$ int $A \subseteq A$;

(ii) semi-open [8] if $A \subseteq c l$ intA and semi-closed if int $c l A \subseteq A$;

(iii) semi-pre-open [1] if $A \subseteq c l$ int $c l A$ and semi-pre-closed if int $c l$ int $A \subseteq A$;

(iv) $\alpha$-open [14] if $A \subseteq$ int $c l$ int $A$ and $\alpha$-closed if $c l$ int $c l A \subseteq A$;

(v) regular open [17] if $A=\operatorname{int} c / A$ and regular closed if $A=c l \operatorname{int} A$.

(vi) Л-оpen [22] if $A$ is a finite union of regular open sets.

The semi-pre-closure(resp. semi-closure, resp. pre-closure, resp. $\alpha$-closure) of a subset $A$ of $X$ is the intersection of all semi-pre-closed (resp. semi-closed, resp. pre-closed, resp. $\alpha$-closed) sets containing A and is denoted by spclA (resp. sclA, resp.pclA, resp. $\alpha c / A)$.

\section{Definition 2.2}

A subset $A$ of a space $X$ is called

(i) generalized closed[9] ( briefly g-closed) if $c l A \subseteq U$ whenever $A \subseteq U$ and $U$ is open.

(ii) regular generalized closed[15](briefly rg-closed) if clA $\subseteq U$ whenever $A \subseteq U$ and $\mathrm{U}$ is regular open.

(iii) $\alpha$-generalized closed[10](briefly $\alpha$ g-closed) if $\alpha c l A \subseteq U$ whenever $A \subseteq U$ and $U$ is open.

(iv) generalized-semi pre-regular-closed [16] (briefly gspr-closed) if spclA $\subseteq U$ whenever $A \subseteq U$ and $U$ is regular-open.

(v) generalized semi-closed [3](briefly gs-closed) if sclA $\subseteq U$ whenever $A \subseteq U$ and $U$ is open.

(vi) Л-generalized closed [5](briefly лg-closed) if $\mathrm{clA} \subseteq \mathrm{U}$ whenever $\mathrm{A} \subseteq \mathrm{U}$ and $\mathrm{U}$ is $\pi$-open.

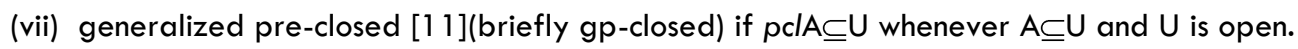

(viii) generalized semi-pre-closed [4](briefly gsp-closed) if spclA $\subseteq$ Uhenever $A \subseteq U$ and

$\mathrm{U}$ is open. 
(ix) $\pi$-generalized pre-closed [7] ( briefly $\pi g p$-closed) if $p c \mid A \subseteq U$ whenever $A \subseteq U$ and $\mathrm{U}$ is $\pi$-open.

(x) generalized pre-regular closed[6]( briefly gpr-closed) if pclA $\subseteq \mathrm{U}$ whenever $\mathrm{A} \subseteq \mathrm{U}$ and $\mathrm{U}$ is regular open.

(xi) weakly generalized closed[13] (briefly wg-closed)if $c l$ int $A \subseteq U$ whenever $A \subseteq U$ and $U$ is open.

(xii) $\pi$-generalized semi-pre-closed[16]( briefly $\pi$ gsp-closed) if spclA $\subseteq U$ whenever $\mathrm{A} \subseteq \mathrm{U}$ and $\mathrm{U}$ is $\pi$-open.

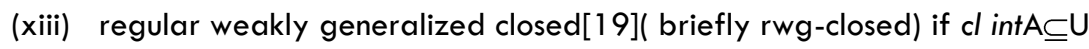
whenever $A \subseteq U$ and $U$ is regular open.

The complements of the above mentioned closed sets are their respective open sets. For example a subset $B$ of a space $X$ is generalized open (briefly g-open) if $X \backslash B$ is g-closed.

\section{Definition 2.3}

A subset $A$ of a space $X$ is called

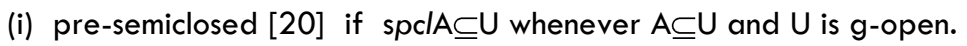

(ii) pre-generalized pre-regular-closed[2] (briefly pgpr-closed) if $p c / A \subseteq U$ whenever $A \subseteq U$ and

$U$ is rg-open.

The complements of the above mentioned closed sets are their respective open sets.

The following lemmas will be useful in sequel.

Lemma 2.4 [2]

If $A$ is semi closed then $p c l(A \cup B)=p c l A \cup p c l B$.

Lemma 2.5 [1]

For any subset $A$ of $X$, the following results hold:

$$
\begin{aligned}
& \text { (i) } \quad \text { sclA }=A \cup \text { int } c l A ; \\
& \text { (ii) } \quad \text { pclA }=A \cup c l \text { intA; } \\
& \text { (iii) } \quad \text { spclA }=A \cup \text { int cl intA. }
\end{aligned}
$$

Lemma 2.6 [18]

If $A$ is semi closed in $X$, then $c l \operatorname{int}(A \cup B)=c l \operatorname{int} A \cup c l$ int $B$.

Lemma 2.7 [6]

If $A$ is regular-open and gpr-closed then $A$ is pre-closed and hence clopen. 


\section{Definition 2.8}

A space $X$ is called extremally disconnected [21] if the closure of each open subset of $X$ is open

\section{Regular pre-semiclosed sets}

Veerakumar[20] introduced pre-semiclosed sets in the year 2002 and Anitha et al.[2] introduced pgpr-closed sets by replacing "spcl" by "pcl" and "g-open" by "rg-open" in the definition of pre-semiclosed sets. In an analog way the regular pre-semiclosed sets are defined by replacing "g-open" by "rg-open". If every rg-open neighbourhood of A contains its semipreclosure, then $A$ is called a regular pre-semiclosed subset. The formal definition of this concept is as follows.

\section{Definition 3.1}

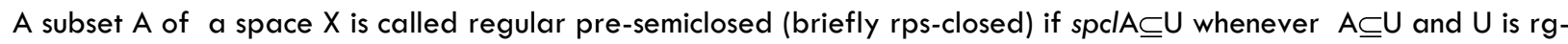
open.

\section{Proposition 3.2}

(i) Every semi-pre-closed set is rps-closed.

(ii) Every pgpr-closed set is rps-closed.

(iii) Every pre-closed set is rps-closed.

(iv) Every $\alpha$-closed set is rps-closed.

(v) Every regular closed set is rps-closed.

\section{Proof}

(i) Let $A$ be a semi-pre-closed set in $X$. Since spclA $=A$, it follows that $A$ is rps-closed.

(ii) Let $A$ be a pgpr-closed set in $X$. Let $A \subseteq U$ and $U$ is rg-open. Since $A$ is pgpr-closed, $p c l A \subseteq U$. Again since spcl $A \subseteq$ pclA, we see that spclA $\subseteq$ U. Therefore $A$ is rps-closed

(iii) follows from (ii) and the fact that every pre-closed set is pgpr-closed .

(iv) follows from (iii) and the fact that every $\alpha$-closed set is pre-closed.

(v) Let $A$ be a regular closed subset of $X$. Let $A \subseteq U$ and $U$ is rg-open. Since $A$ is regular-closed, $A=c l \operatorname{int} A$. $c l \operatorname{int} A$ $\subseteq \mathrm{U}$ and $\mathrm{U}$ is rg-open. int $\mathrm{cl}$ int $\mathrm{A} \subseteq$ int $\mathrm{U} \subseteq \mathrm{U}$ and $\mathrm{U}$ is rg-open.

$\mathrm{A} \cup$ int $c l$ intA $\subseteq \mathrm{A} \cup \mathrm{U} \subseteq U$ and $U$ is rg-open. spclA $\subseteq U$ whenever $A \subseteq U$ and $U$ is rg-open. Therefore $A$ is rpsclosed.

The reverse implications are not true as shown in Example 3.4 and Example 3.5.

\section{Proposition 3.3}

(i) Every rps-closed set is pre-semi-closed.

(ii) Every rps-closed set is gspr-closed.

(iii) Every rps-closed set is gsp-closed. 


\section{Proof}

(i) Let $A$ be a rps-closed subset of a space $X$. Let $A \subseteq U$ where $U$ is g-open. Since every g-open set is rg-open and since $A$ is rps-closed, by Definition 2.3(i), A is pre-semi-closed.

(ii) Let $A$ be a rps-closed subset of a space $X$. Let $A \subseteq U$ and $U$ is regular-open. Since every regular-open set is rgopen and since $A$ is rps-closed, by Definition 2.2 (iv), spclA $\subseteq U$.

Therefore $A$ is gspr-closed.

(iii) Let $A$ be a rps-closed subset of a space $X$. Let $A \subseteq U$ and $U$ is open. Since every open set is $g$-open and since every g-open set is rg-open, spclA $\subseteq \mathrm{U}$ and hence $A$ is gsp-closed.

The reverse implications are not true as shown in Example 3.4.

\section{Example 3.4}

Let $X=\{a, b, c, d\}$ with topology $\tau=\{\varphi,\{a\},\{b\},\{a, b\},\{b, c\},\{a, b, c\}, X\}$. Then

(i) $\{a, b, d\}$ is rps-closed but not semi-pre-closed.

(ii) $\{b, d\}$ is pre-semiclosed but not rps-closed .

(iii) $\{a\}$ is rps-closed but not pgpr-closed set.

(iv) $\{a, b\}$ is gspr-closed but not rps-closed.

(v) $\{b, c\}$ is rps-closed but not pre-closed.

(vi) $\{b, c\}$ is rps-closed but not $\alpha$-closed.

(vii) $\{b, d\}$ is gsp -closed but not rps-closed.

\section{Example 3.5}

Let $X=\{a, b, c\}$ with topology $\tau=\{\varphi,\{a, b\}, X\}$. Then $\{a\}$ is rps-closed but not regular-closed.

The concept of rwg-closed, wg-closed, gpr-closed, $\pi$ g-closed, $\pi$ gp-closed, gp-closed, rg-closed, $\alpha$-closed sets are independent with the concept of rps-closed as shown in the following example.

\section{Example 3.6}

Let $X=\{a, b, c, d\}$ with topology $\tau=\{\varphi,\{a\},\{b\},\{a, b\},\{b, c\},\{a, b, c\}, X\}$.

(i) $\{a\}$ is rps-closed but not rwg-closed and $\{a, b\}$ is rwg-closed but not rps-closed.

(ii) $\{a\}$ is rps-closed but not wg-closed and $\{b, d\}$ is wg-closed but not rps-closed.

(iii) $\{a\}$ is rps-closed but not gpr-closed and $\{b\}$ is gpr-closed but not rps-closed.

(iv) $\{a\}$ is rps-closed but not $\pi \mathrm{g}$-closed and $\{b, d\}$ is $\pi \mathrm{g}$-closed but not rps-closed.

(v) $\{a\}$ is rps-closed but not $\pi \mathrm{gp}$-closed and $\{b, d\}$ is $\pi \mathrm{gp}$-closed but not rps-closed

(vi) $\{b, d\}$ is gp-closed but not rps-closed and $\{a, b, d\}$ is rps-closed but not gp-closed.

(vii) $\{a, b\}$ is rg-closed but not rps-closed and $\{a\}$ is rps-closed but not rg-closed.

(viii) $\{a\}$ is rps-closed but not $\alpha \mathrm{g}$-closed and $\{b, d\}$ is $\alpha g$-closed but not rps-closed.

The concept of g-closed and rps-closed sets are independent as shown in the following example. 


\section{Example 3.7}

Let $X=\{a, b, c, d\}$ with $\tau=\{\varphi,\{a\},\{a, b\}, X\}$. Then $\{b\}$ is rps-closed but not $g$-closed and $\{a, c\}$ is g-closed but not rpsclosed.

The concept of gs-closed and rps-closed sets are independent as shown in the following example.

\section{Example 3.8}

Let $X=\{a, b, c\}$ with topology $\tau=\{\varphi,\{a, b\}, X\}$. Then $\{a\}$ is rps-closed but not gs-closed.

From Example 3.6 we see that $\{b, d\}$ is gs-closed but not rps-closed.

Thus the above discussions lead to the implication diagram given at the end. In this diagram by "A $\rightarrow$ B" we mean A implies B but not conversely and

"A $\longrightarrow$ " means $A$ and $B$ are independent of each other.

The Union and intersection of two rps-closed sets need not be rps-closed as shown in the following example.

\section{Example 3.9}

$$
\text { Let } X=\{a, b, c, d\} \text { with } \tau=\{\varphi,\{a\},\{b\},\{a, b\},\{b . c\},\{a, b, c\}, X\} \text {. Then } A=\{a\} \text {, }
$$

$B=\{b, c\}$ and $C=\{a, b, d\}$. Here $A$ and $B$ are rps-closed but $A \cup B=\{a, b, c\}$ is not rps-closed. Also $B$ and $C$ are rpsclosed but $B \cap C=\{b\}$ is not rps-closed.

\section{Theorem 3.10}

If $A$ is regular-open and $A$ is gpr-closed then $A$ is (i) rps-closed (ii) gspr-closed.

\section{Proof}

Follows from Lemma 2.7 and Diagram 1

\section{Theorem 3.11}

If $A$ is semi-closed then $\operatorname{spcl}(A \cup B)=\operatorname{spcl} \cup \cup \operatorname{spcl} B$.

\section{Proof}

Suppose A is semi-closed. By Lemma 2.5(iii),

$\operatorname{spcl}(A \cup B)=(A \cup B) \cup \operatorname{int} c l i n t(A \cup B)$.

$\operatorname{spcl}(A \cup B)=(A \cup B) \cup \operatorname{int}[c l \operatorname{int} A \cup c l$ int $B]$ by applying Lemma 2.6.

$=(A \cup B) \cup[\operatorname{int} c l$ int $A \cup \operatorname{int} c l \operatorname{int} B]$

$=(A \cup \operatorname{int} c l \operatorname{int} A] \cup[B \cup \operatorname{int} c l$ int $B]$

$=[\mathrm{A} \cup \operatorname{int} \mathrm{cl} \operatorname{int} \mathrm{A}] \cup[\mathrm{B} \cup \operatorname{int} \mathrm{cl} \operatorname{int} \mathrm{B}]$

$\operatorname{spcl}(A \cup B)=\operatorname{spcl} \cup \cup \operatorname{spclB}$. 


\section{Theorem 3.12}

Let $A$ and $B$ be rps-closed sets and let $A$ be semi-closed. Then $A \cup B$ is rps-closed.

Proof

Let $A \cup B \subseteq U$ and $U$ be rg-open. Then $A \subseteq U$ and $B \subseteq U$. Since $A$ and $B$ are rps-

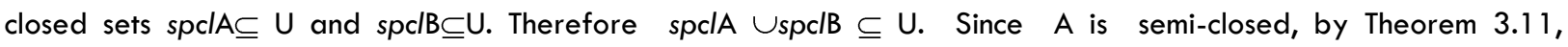
$\operatorname{spcl}(A \cup B) \subseteq U$. Hence $A \cup B$ is rps-closed.

\section{Theorem 3.13}

If a set $A$ is rps-closed then, spclA \A does not contain a non empty rg-closed set.

\section{Proof}

Suppose that $A$ is rps-closed. Let $F$ be a rg-closed subset of spclA \A. Then

$F \subseteq$ spcl $A \cap(X \backslash A) \subseteq X \backslash A$ and so $A \subseteq X \backslash F$. But $A$ is rps-closed. Since $X \backslash F$ is rg-open,

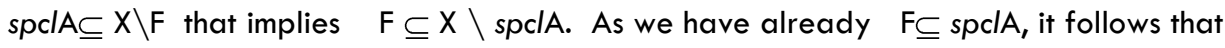

$\mathrm{F} \subseteq \operatorname{spclA} \cap(\mathrm{X} \backslash \operatorname{spclA})=\varnothing$. Thus $\mathrm{F}=\varnothing$. Therefore spclA \A does not contain a non empty rg-closed set

\section{Theorem 3.14}

Let $A$ be rps-closed. Then $A$ is semi-pre-closed if and only if spclA $\backslash A$ is rg-closed.

\section{Proof}

If $A$ is semi-pre-closed then $\operatorname{spcl}(A)=A$ and so $\operatorname{spcl} \backslash \backslash A=\varnothing$ which is $\mathrm{rg}$-closed.

Conversely, suppose that spclA \A is rg-closed. Since $A$ is rps-closed,

by Theorem 3.13, spclA $\backslash A=\varnothing$. That is spclA $=A$ and hence $A$ is semi-pre-closed.

\section{Theorem 3.15}

If $A$ is rps-closed and if $A \subseteq B \subseteq$ spclA then

(i) $B$ is rps-closed

(ii) $\operatorname{spc} / \mathrm{B} \backslash \mathrm{B}$ contains no non empty rg-closed set.

\section{Proof}

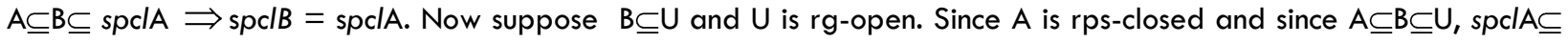
$U$ that implies $s p c / B \subseteq U$. This proves (i). Since $B$ is rps-closed, (ii) follows from Theorem 3.13.

\section{Theorem 3.16}

For every point $\mathrm{X}$ of a space $\mathrm{X}, \mathrm{X} \backslash\{\mathrm{x}\}$ is rps-closed or rg-open.

\section{Proof}

Suppose $X \backslash\{x\}$ is not rg-open. Then $X$ is the only rg-open set containing $X \backslash\{x\}$. This implies $\operatorname{spcl}(X \backslash\{x\}) \subseteq X$. Hence $X \backslash\{x\}$ is rps-closed set in $X$.

Theorem 3.17

Suppose $A$ is rg-open and $A$ is rps-closed. Then $A$ is semi-pre-closed. 


\section{Proof}

Since $A$ is rg-open and since $A$ is rps-closed,$A \subseteq A \Rightarrow$ spclA $\subseteq A$. This proves the theorem.

\section{Theorem 3.18}

Let $A$ be rps-closed and $c l$ int $A$ be open. Then $A$ is pgpr-closed.

Proof

Let $A \subseteq U$ and $U$ be rg-open. Since $A$ is rps-closed, spclA $\subseteq U$. By Lemma 2.5 (iii)

$\mathrm{A} \cup$ int $\mathrm{cl}$ int $\mathrm{A} \subseteq \mathrm{U}$ that implies $\mathrm{A} \cup \mathrm{cl}$ int $\mathrm{A} \subseteq \mathrm{U}$. Applying Lemma 2.5 (ii) pclA $\subseteq \mathrm{U}$.

Therefore A is pgpr-closed.

\section{Corollary 3.19}

In an extremally disconnected space $X$, every rps-closed set is pgpr-closed.

\section{Proof}

In an extremally disconnected space $X, c l$ intA is open for every subset A of $X$. Then the Corollary follows directly from Theorem 3.18.

\section{Diagram 1}

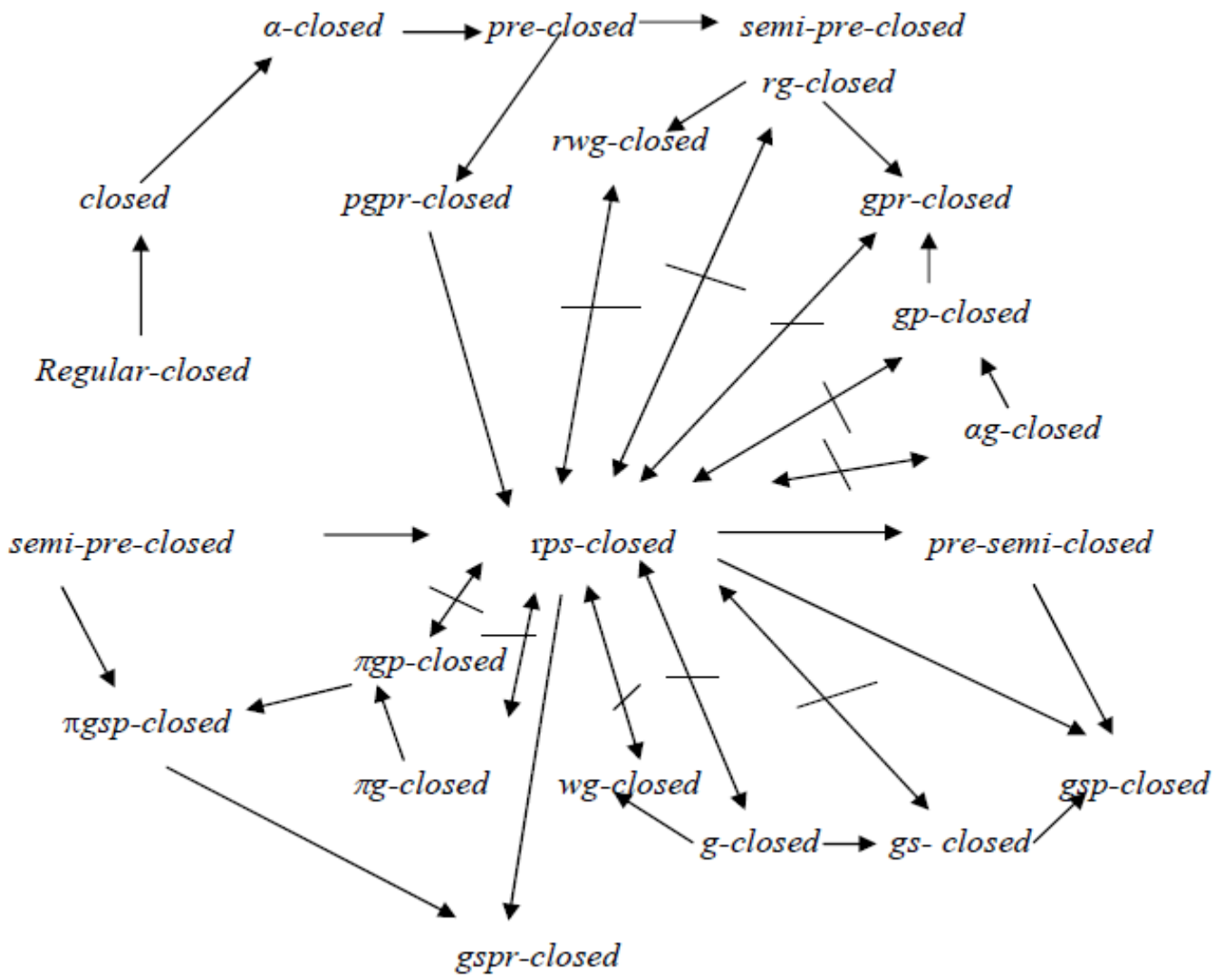




\section{References}

[1] Andrijevic D (1986) Semi-preopen sets. Mat Vesnik 38: 24-32.

[2] Anitha $M$ and P Thangavelu (2005) On Pre-Generalized Pre-Regular-Closed sets. Acta Ciencia Indica 31 M (4): $1035-1040$.

[3] Arya S and T Nour(1990) Characterizations of s-normal spaces. Indian J. Pure Appl. Math 21: 717-719.

[4] Dontchev J (1995) On generalizing semi-pre-open sets. Mem Fac Sci Kochi Univ Ser A Math. 16: 35-48.

[5] Dontchev J and T Noiri (2000) Quasi-normal spaces and Mg-closed sets. Acta Math. Hungar 89 (3): 21 1-219.

[6] Gnanambal Y(1997) On generalized pre regular closed sets in topological spaces. Indian J. Pure Appl. Math. 28

(3): 351-360.

[7] Janaki C (1999) Studies on $\pi g \alpha$-closed sets in topology. Ph.d Thesis. Bharathiar university Coimbatore, India.

[8] Levine N (1963) Semi-open sets and semi continuity in topological spaces. Amer. Math. Monthly 70: 36-41.

[9] Levine N (1970) Generalized closed sets in topology. Rend. Circ. Mat. Palermo 19 (2): 89-96.

[10] Maki H, R Devi and K Balachandran (1994) Associated topologies of generalized $\alpha$-closed Sets and $\alpha$ generalized closed sets. Mem. Fac. Sci. Kochi Univ. (Math) 15: 51-63.

[11] Maki H, J Umehara and T Noiri (1996) Every topological space is pre-T1/2. Mem Fac Sci Kochi Univ Ser.A, Math 17: 33-42.

[12] Mashhour AS, ME Abd El-Monsef and SN El-Deeb (1982) On precontinuous and weak Precontinuous functions. Proc. Math. Phys. Soc . Egypt 53: 47-53.

[13] Nagaveni N (1999) Studies on generalizations of homeomorphisms in topological spaces. Ph.D Thesis. Bharathiar University, Coimbatore, India.

[14] Niastad O (1965) On some classes of nearly open sets. Pacific J. Math.15: 961-970.

[15] Palaniappan N and KC Rao (1993) Regular generalized closed sets. Kyungpook Math. J 33: 211 -219.

[16] Sarsak MS and N Rajesh (2010) $\pi$-Generalized semi-pre-closed sets. International Mathematical Forum 5(12): 573-578.

[17] Stone M (1937) Application of the theory of Boolean rings to general topology. Trans. Amer. Math. Soc 41 : 374-481.

[18] Thangavelu P and KC Rao (2002) p-sets in topological spaces. Bulletin of Pure and Applied Sciences 21E (2): $341-355$.

[19] Vadivel A and K Vairamanickam (2009) rga-closed sets and rg $\alpha$-open sets in topological Spaces. Int J Math Analysis 3 (37): 1803-1819.

[20] Veerakumar MKRS (2002) Pre-semi-closed sets. Indian J. Math 44 (2): 165-181.

[21] Willard S (1970) General Topology. Addison Wesley.

[22] Zaitsav V(1968) On certain classes of topological spaces and their bicompactifications. Dokl. Akad. Nauk SSSR 178: $778-779$. 